\title{
Optimal Electric Vehicle Charging Control Strategy Powered by Grid-Linked Hybrid PV-Wind-Battery Renewable Energy System
}

\author{
Adel Elgammal, Tagore Ramlal
}

\begin{abstract}
An advanced model is proposed for grid connectivity of an interconnected network consisting of a charging station for electric automobiles. To automate the discharge procedure of charging/ the battery energy storing system, a wind network, the photovoltaic system, and the battery energy storing system is developed to efficiently increase the consumption degree of solar and wind energy sources and create renewable inner-city capacity. On the basis of DC bus architecture, the power design was planned such that buffered storage systems and renewable energy resources can be incorporated. The proposed optimal control algorithm uses the Swarm Optimization Algorithm consists of Multi-Objective Particle, developed for electric vehicles charging or discharge behaviors to minimize the overall actual energy loss and increase the integration of EVs with power networks due to the efficiency and economy of network activity, taking into account the economic issue and the satisfaction of consumers, the voltage limits and the parking availability pattern. To test the proposed EV charging strategy, simulation studies based on efficiency, and assessed major energy fluxes within the device. Energy management approaches have also been developed to optimize the power requirements and charging times of various electric vehicles. Results suggest that proposed model will substantially reduce the power grid's operational costs while meeting the charging criteria of the customer. Improved performance on global search capabilities is also checked, as is the desired outcome of enhanced particle swarm optimization algorithm. The findings show that the new approach is in a position to prepare EV charging times optimally, taking into account electronic knowledge and uncertainty.

Keywords: Electric vehicles, Photovoltaic systems, Energy storage, Adaptive charging control algorithm, charging station, energy management system, and multi-objective particle swarm optimization.
\end{abstract}

\section{INTRODUCTION}

With a great amount of PHEVs integrated into the distribution grid, a growing number of on-board batteries need to be charged via the infrastructure, such as with dedicated charging stations in parking lots. Transport sector electrification has the ability to stimulate future demand for

Revised Manuscript Received on August 15, 2020.

* Correspondence Author

Adel Elgammal*, the University of Trinidad and Tobago UTT, Utilities Engineering, Point Lisas Campus, Trinidad and Tobago. E-mail: adel.elgammal@utt.edu.tt

Tagore Ramlal, the University of Trinidad and Tobago UTT, Utilities Engineering, Point Lisas Campus, Trinidad and Tobago. E-mail: Tagore.Ramlal@utt.edu.tt

(c) The Authors. Published by Blue Eyes Intelligence Engineering and Sciences Publication (BEIESP). This is an open access article under the CC BY-NC-ND license (http://creativecommons.org/licenses/by-nc-nd/4.0/) transport with high energy efficiency, energy independence and low environmental impacts, such as greenhouse gas releases [1]. The rise of electric vehicles (EVs) has raised the need for charging locations that have already been challenged with the question of access to the power grid. A plan to link electric vehicle charging stations to local renewable energy has been introduced to decrease the rising need and pressure on the national power network owing to the exponential rise in EVs. [2]. Wind / photovoltaic / battery energy storage on a small scale strikes the energy equilibrium among local manufacture and depletion by storage and optimum distribution. When needed to charge EVs, it will connect with the power grid using the battery energy storage network. It reduces the outcome of charging on the power network and increases the performance of electricity production. Once attached to the electricity control system, the current transfer between the power network and the electric vehicle will be bidirectional; the electric vehicle input is drain on the network, while the electric vehicle discharge is a power resource [3-4]. Research into grid-connected sustainable energy technologies and the construction of charging infrastructures for electric vehicles directly linked to the generation of renewable energy from the micro grid are becoming very necessary to increase the capacity for renewable energy absorption from the grid [5]. Furthermore, when linked to charging stations, the electrical and plug-in hybrid car battery systems pose a major benefit for main network in the incorporation / connection partnership using renewable energy resources. By the standpoint, the electrical machinery production is presented with problems surrounding the eventual incorporation of electric vehicles into power network. Performance of electricity, overloading of lines and networks, elevated power shortages, demand supply inequite and volatility are amongst the utmost severe influences levied by EVs [6-7]. An EV should be called a flexible charge payable during the day, rather than a fixed fee schedule [8]. In terms of flattening the load curve on large substation transformers, the versatility of $\mathrm{EV}$ demand will enhance the performance of power grids, if peak savings facilities, minimized power network failures, decreased transformer and line ageing and increased clean energy integration as well as financial support [9]. EVs will be charged once the user reaches at the charging station without an appropriate EV charging device, leading to unnecessary spikes in peak power demand and reducing the available grid capacity [10]. Neither can the electric power network respond to these requests. Nonetheless,

Published By:

Blue Eyes Intelligence Engineering and Sciences Publication

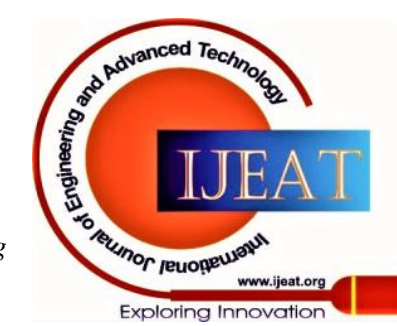


probable difficulties for example unexpected topmost demand or unexpected overloading may be expected and modified using an adaptive EV battery charging program. In Demand-Side Management and adopted by the EV aggregator to boost the distribution network performance, EV charging optimization is a request-response approach that must be implemented [11]. Minimum cost of charging was achieved by optimally planning the charging power and time of EVs in [12] treating congestion management as a device constraint. Coordinated enhanced charging control strategies of many EVs are considered for public charging stations without having an effect on the power grid to discharge (and charge) the batteries over their entire lifetime viewpoint [13]. The charging power is optimized using a globally optimal schedule to decrease the overall expense of all the EVs charging and discharging throughout the daytime [14]. Considering the regular and real-time markets based on heuristic algorithms, an optimisation problem with the scheduling of EV charging with energy storage was studied [15]. A system has been introduced to simplify charging and discharge of EVs in power spot markets with specific travel trends for car fleets [16], ignoring the loss of grid network. A smart charging algorithm was implemented [17], which provides consumers and aggregators major financial benefits for various battery maintenance costs and increased device versatility. It is also undergoing peak load declines, minus grid management infrastructure prices. To minimize charging costs in a competitive market focused on the basis of Time-of-Use (TOU), a heuristic approach for solving an automated EV load management model was implemented [18]. To minimize pressure on the power network and satisfy the requirements of the individual car owners, a novel strategy was suggested [19] for planning electric vehicle charging without electricity grid constraints. Increased demand for electric vehicles is causing considerable concern about the influences on the power grid. So, if at the same time a large number of EVs connect to the grid, a wide range of network impacts will occur. The electric vehicle charging prototypes are checked for the time character of the connection based on the specific way the electric vehicles are linked to power grid. Numerous findings have looked at the energy storage network to improve grid efficiency in the construction of PV supported electric vehicle charging stations. To optimize self-consumption and autonomy, the technological efficiency of small Scale Wind / Photovoltaic / Battery Store Power in a campus micro grid was evaluated [20]. An optimisation model was proposed to implement the energy storage network to reduce the operational costs of EV charging stations using electricity prices in real time [21]. A PSO-based optimisation model with a financial model was used to scale the PV and battery power storage network in battery storage / electric automobile charging location / photovoltaic connected to a grid [22]. The genetic algorithm program was proposed for optimum PV system design for an isolated photovoltaic / battery energy storage / charging place for electric automobiles [23] and for construction of an EV high-speed charging station, comprising the amount of chargers, the connected renewable energy and energy storing capacity and a contract grid power [24]. An energy managing structure was developed to adjust the energy flow in photovoltaic/battery energy storage/ charging station for electric vehicles between PV, battery power storage and grid [25]. A number of studies merged PSO and Multi-agent method to achieve rapid convergence and increase results accuracy by combining agents and PSO search mechanism [26]. This paper proposed a new configuration of EV charging to fill current holes, such as the need to consider practical driving habits and the various charging functions. The proposed charging solution optimizes the charging of electric vehicles in power grid, taking into account both the electric vehicles owners and power network. An design model will be proposed to decide the optimum scale of the solar, photovoltaic and battery energy storage networks in the grid-connected wind/photovoltaic/battery energy storage/electric vehicle charging stations network and to analyze the correct energy management technique, such as charging and discharge patterns for the battery energy storage network and the power plant connection. (MOPSO was used to obtain higher convergence time and more reliable performance aiming to resolve the proposed problem. The optimal target prototype requires the degree of comfort of the EV holder, the smoothness of the regular charging curve of the generator, the reduction of the deficit of active power transfer and modification of power network regulator tools. The prototype also takes into account network limits, communication capability restrictions, resource limitations for charging and discharging, and the charging status of EVs (SOCs) limits.

\section{THE PROPOSED CONFIGURATION FOR ELECTRIC VEHICLE CHARGING STATION / BATTERY ENERGY STORAGE / WIND/ PHOTOVOLTAIC}

Micro grid technology has been developed and applied for simulation research for the fast recharging of electric vehicles with the goal of researching and assessing the operational features of the DC-based recharging location with a buffered storing network and an electric vehicle battery-operated pack. The DC micro grid consists primarily of a Wind / PV hybrid network, an EV turbine, a battery-powered storage facility, a grid-connected network and a micro grid energy distribution system as seen in Fig.1. This consists of a combination of wind turbines and PV as a source of electricity. The DC / DC converter makes use of the boost circuit to power the PV modules while a three-phase rectifier links the adjustable speed wind turbine to the Stable Magnet Synchronous Generator. Where a grid interface inverter and the RL filter are required to transfer the induced power to the grid, the two energy sources are linked to the DC-link voltage in parallel. The EV charging unit consists of a DC converter and an EV motor, a motor amplifier and a controllable current. Recently, the battery was also filled with continuous fuel. The charging power and the charging speed determine the Charge time in the constant charging mode. The energy storage unit consists of the lithium-ion battery and the DC / DC transducer. The energy storage system facilitates load shifting, the dissolved Wind / PV hybrid system, and the power fluctuation smoothing feature. The grid-connected network consists mainly of the AC / DC bi-directional inverter and the control transformer. In comparison, the key drawback of the use of solar and wind power is the fossil fuel use and removal in greenhouse gas emissions. Nevertheless,

Published By:

Blue Eyes Intelligence Engineering and Sciences Publication (C) Copyright: All rights reserved.

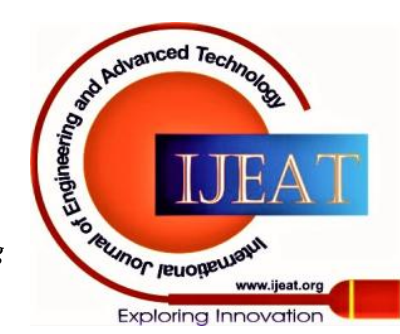


these types of energy resources cannot be shipped. And in order to ensure their successful alignment with the main grid, their intrinsic volatility and instability are deemed to be critical problems that need to be resolved.

Particularly, fluctuations in electrical power associated with solar generation structures may be calculated on a preliminary basis. Because the motion of the sun is understood, but with very rapid adjustments, the unexpected occurrence of clouds and/or other reasons the incompatibility that limit the power generated by PV systems. Power variations in wind generation structures are usually time consuming and less stable relative to PV generation systems, with substantial shifts in energy output as a result of wind gusts. In fostering the convergence of electric vehicle and (RESs with main grid) above-mentioned problems can be adequately solved by energy storage technologies in the smart grid climate. Alternatively, by storing the excess electrical energy generated by RESs, PEV battery packs can assist the main grid during low power demand cycles. In this situation, the central grid delivers power flow to on-load vehicles that can compete with the utility market's frequency and voltage regulatory services. In comparison, to assist the main grid with ancillary services also mentioned to as vehicle-to - grid facilities, electrical energy kept in battery packs of PEVs may be used, which often act as well-organized peak power and spinning resources. In this latter scenario, the use of bi-directional on-board or off-board battery chargers and PEVs communicate with the key grid as power users and as distributed generation systems, is appropriate for this reason. Bidirectional V2 G networks should offer ancillary facilities of superior quality to the grid. Unfortunately, the economic advantages of this shared relationship among PEVs and the grid are offset by the necessity to introduce different security procedures, for example anti-isolation steps, and rising costs of bi-directional charging systems for mobile devices.

During operation, the grid-connected network transfers electricity between the power system and the DC micro grid, this holds the DC bus voltage constant. The operation of each node within the DC micro grid monitored and controlled by the micro network energy management program. The hybrid Wind / PV battery, the energy storage system or the data-based distribution grid generate the energy management system tracks the charging power of EV and charging power. The micro-grid energy management program helps to achieve dissolved PV power, maximize EV charging and balancing of micro grids. This idea is built on a network of direct coordination among paying vehicles and Grid System Operator. Under this system, via direct lines of communication, each vehicle is operated by the GSO as a single deterministic power resource. Additionally, the owner of the vehicle may share ancillary services with the GSO, the availability of which terminates when vehicle departs the charging point. A very basic scheme was illustrated by the direct architecture for handling V2 G operations. In addition, a broad diffusion of high-bandwidth communication lines provided by the geographically dispersed existence of V2 G services was entailed by continuous communications between GSO and on-load vehicles. Long-standing forecasts also need to take into account the anticipated rise in the amount of vehicles involved and disengaged for simultaneous interaction with GSO, depending on possible market penetration scenarios. In this sense, it is assumed that the GSO will continue to monitor the contract status, contact information, owner conditions and level of payments for each PEV.

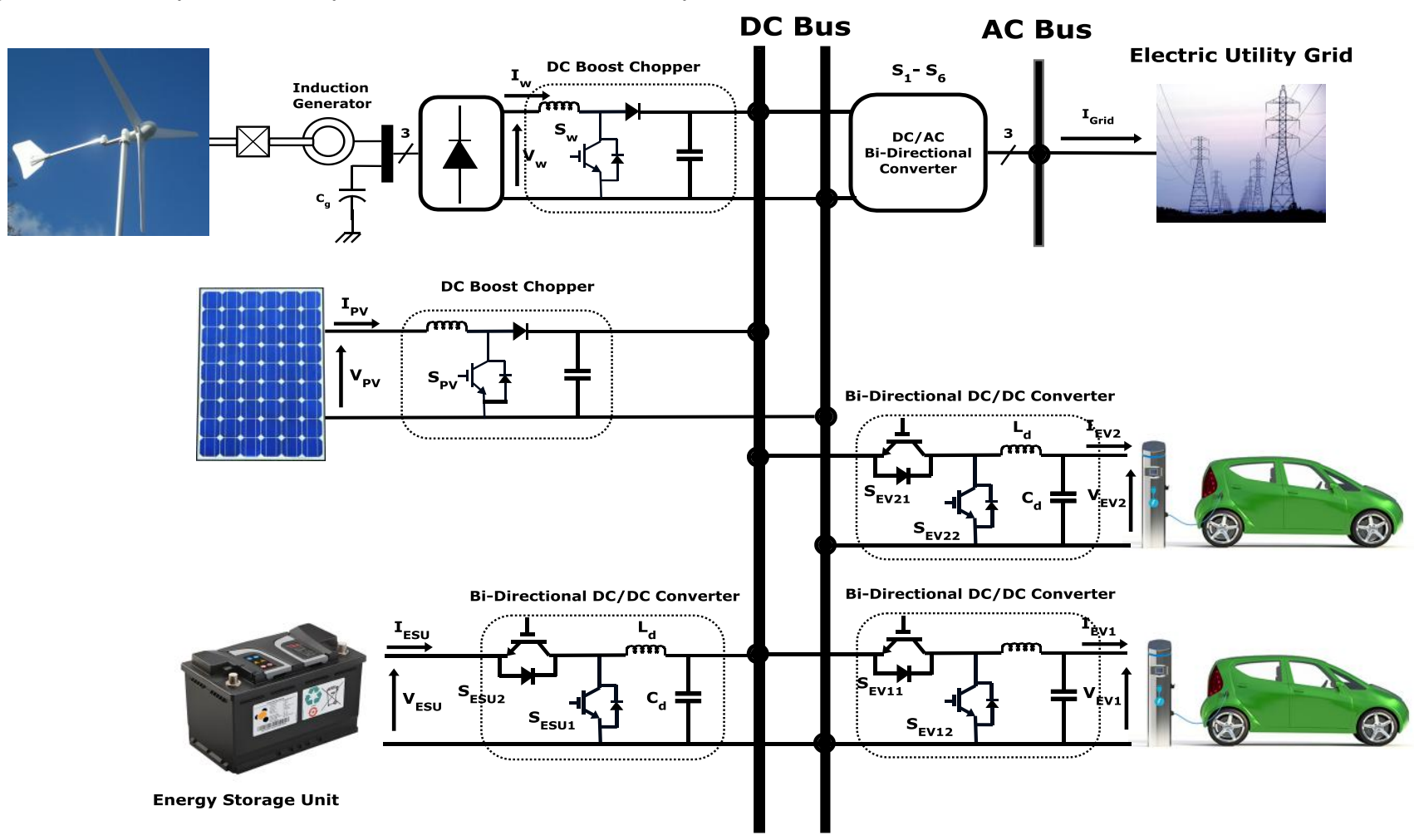

Figure 1. Block scheme of the structure of the Electric automobile charging station.

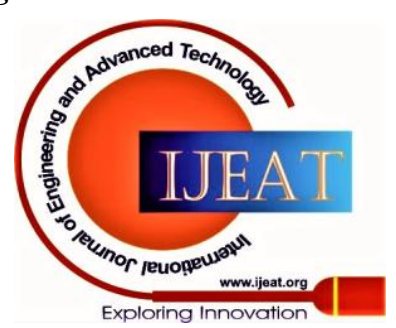




\section{THE PROPOSED REAL-TIME ENERGY MANAGING PROCEDURE}

To optimize the relationship between several energy resources, the real-time energy management scheme is needed, such as wind, photovoltaic and battery storage, in line with load demand and the need for EV charging. In this article, to charge the EV during the day without interrupting the demand for ac power, energy storage scheme is utilized that uses the MOPSO optimization strategy. The full micro-grid system was split into following operating modes. The power flow direction for optimal use of the available resources is monitored by operating modes. The suggested operating modes are concise as follows: Mode 1: If the PV hybrid power structure / Wind is adequate to satisfy need for EV charging, the on-board charging is carried out completely by the Wind / PV hybrid power structure. The combination PV power structure / Wind will charge EV individually without reaching the grid. Mode 2: When the Wind / PV Hybrid Power Unit is completely unavailable but ESU has sufficient energy to satisfy the need for EV charging on its own, this mode is allowed. In the lack of a Wind / PV hybrid power plant, the plant load is minimized. Mode 3: The EV will be powered directly from the grid while the Wind / PV hybrid system and the ESU were absolutely unable to produce any electricity at all. Mode 4: If no electric vehicles is to be charged and the Wind / PV hybrid power system is fewer than or same to equivalent ESU SOC, then the entire Wind / PV hybrid power system will go to ESU. This reduces grid dependency by storing ESU electricity for future usage. Mode 5: When there is no EV to be powered, so that when the Wind / PV hybrid power system produces electricity ESU has already exceeded the full SOC cap, all capacity is sold to the grid. Mode 6: The grid provides electricity to the local market while the Hybrid Wind / PV surplus power structure is insufficient to fulfil the need for the AC fee.

\section{THE EV CHARGING OPTIMIZATION CONTROL STRATEGY}

Over the past few years a variety of charging solutions have been built which can be divided into subsequent groups. Irrational charging schemes showing EV charging only continue while they're plugged in house. Conventional structured charging systems demonstrating the charging cycle was phased out to reduce the times of peak demand. A smart charging scheme is built with a sophisticated algorithm to boost power grid operation, showing the G2V charging scheme. V2G-G2V charging systems which indicate charging and discharge of electric vehicle batteries are managed to optimize the power structure output. This post touches on the right method of EV charging to flatten transport network load curve. It further tackles high demand crashing and technical network drawbacks. To accomplish this objective, it should be borne in mind that entire electric vehicle load specifications must be met in accordance with the parking patterns and technical system limitations, for instance thermal cable restricts and bus voltage breaches. The 24 hour fee requires a local network model and low voltage network topology. It is used as data input for the optimisation problem. The terms under which the decision was taken were the allowance for EV parking which could be available at any time for 24 hours. The key reason of the electric vehicle charging controller system is to automate the EV charging network with micro grid energy storage to boost performance and overall charges. The general design of the EV Charging Control System (Fig. 2) displays the flow map for the standard EV charging cycle in the car park. This can be shown, as the EV arrives, details of the battery power and initial charging status (SOC) can be collected. In the meanwhile, the driver of the EV decides which maximum SOC will be achieved before reaching the car park. The battery size and the initial and desired SOCs will then be used to calculate the amount of electricity the $\mathrm{EV}$ requires to charge. In the next step, a suitable charging facility for the EV is chosen on the basis of the necessary amount of energy and departure time, and a charging control model is run to achieve an ideal charging plan.

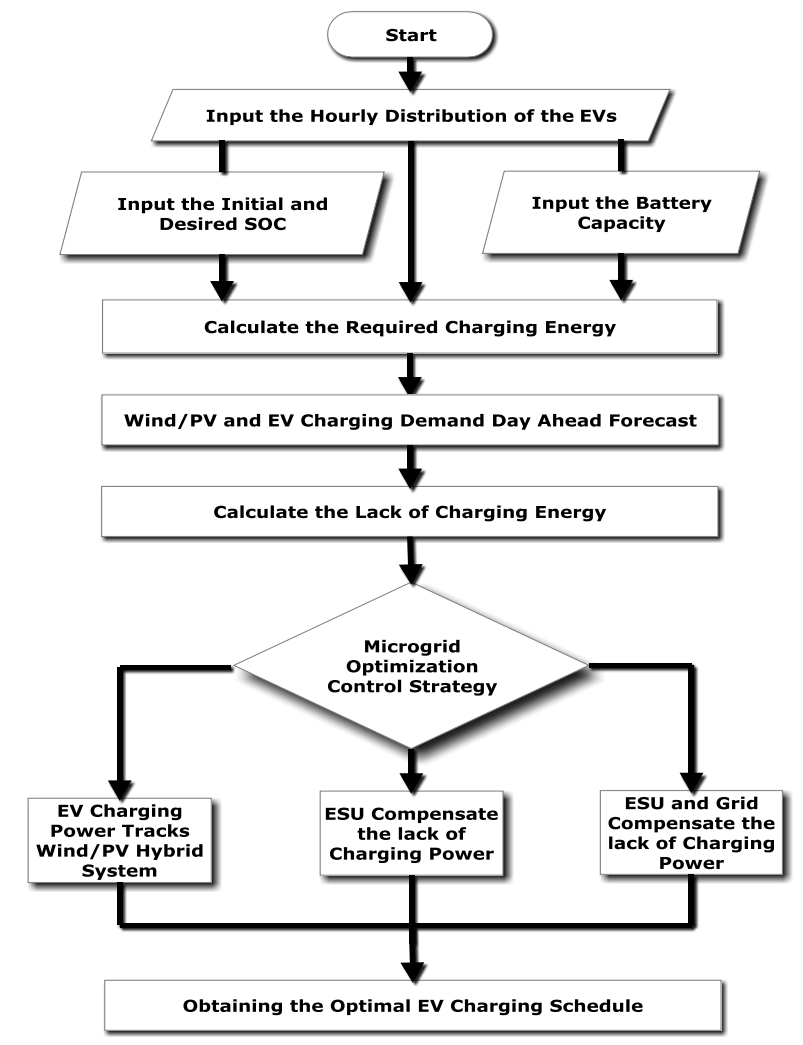

Figure 2. The flow diagram of the optimization control plan of EV charging

\section{SMART CHARGING SCHEME: PROBLEM FORMULATION}

The key characteristics of the electric vehicle batteries are the charging period, the power demand and the charging volume. Data on preliminary charging state of electric automobile batteries is required for each 24-hour period of the day for planning and scheduling purposes. In this article, SOC of every battery shall be assessed to be between the average (SOCmax) and the minimum (SOCmin) rates and the optimal scheduling shall be carried out by means of the following information from the charging location: contact period; primary charging state; real charging state; final charging status; vehicle kind and consumer charging mode.

Published By:

Blue Eyes Intelligence Engineering and Sciences Publication

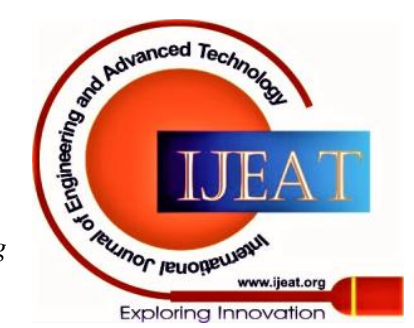


In addition, the efficiency of battery charging is considered to be 90 per cent in the grid's EV energy demand.

PEV pays for each of the charging times before the target level of charge ( SOC) has been achieved.:

$S O C=S O C_{\text {initial }}+\sum_{h=1}^{H} \operatorname{SOC}(h)$

Where ' $\mathrm{H}$ ' is last hourly cycle of charge of the battery and SOC(h) is state of charge at any moment.

Wind / Battery Power Storage / Photovoltaic/ Electric Automobile Charging Station will obey the energy balance law in grid-connected mode, which ensures that the electricity usage is proportional to the total output as follows:

$P_{\text {Grid }}(t)+P_{P V}(t)+P_{\text {Wind }}(t)+P_{\text {dis }}(t) \times\left[\frac{\mu_{2}(t)}{\eta_{\text {dis }}}\right]=P_{c h}(t) \times\left[\eta_{c h} \times \mu_{1}(t)\right]+P_{\text {loads }}(t)$

Where PGrid(t) is the power produced by the distribution grid that can be either negative or positive. In the afore mentioned relation, the electricity generated by the loads plus the electricity added to the Battery Power Storage System is equivalent to energy provided via Wind / PV hybrid device and the power grid plus the power released by the battery at every time of the year. $P_{c h}(t)$ and $P_{d i s}(t)$ signify the charging and discharging power at time $t$, correspondingly; the charging and discharge efficiencies are $\pi \mathrm{ch}$ and ÿdis. $\mu 1(\mathrm{t})$ and $\mu 2(\mathrm{t})$ apply respectively to the "load" and "discharge" phrases. Charging and discharge states are not permitted to work on both states at any time simultaneously, and are defined as:

$$
\begin{aligned}
& \mu_{1}(t)+\mu_{2}(t)=[0,1] \\
& \mu_{1}(t)=[0,1] \\
& \mu_{2}(t)=[0,1]
\end{aligned}
$$

The charging rate of an individual battery is coordinated during the charging period according to the optimal objective functions and charger, battery, and power network constraints. The goal functions consist of three goals for maximizing the charging and unloading actions of a great amount of EVs linked to the power grid, which are defined in detail as follows.

The annual electricity costs are generated through the exchange of energy with the utility grid. If the Wind / PV hybrid network delivers electricity to the central grid, the output is negligible, which ensures the network is competitive. In the opposite, as the machine imports power from the energy grid, the cost of electricity is low. Consequently, the first objective function is to minimize the electricity that is supplied from the grid:

$\min f_{1}=\sum_{t=1}^{T}\left(-P_{P V}(t)-P_{\text {Wind }}(t)-P_{\text {dis }}(t) \times\left[\frac{\mu_{2}(t)}{\eta_{\text {dis }}}\right]+P_{c h}(t) \times\left[\eta_{c h} \times \mu_{1}(t)\right]+P_{\text {loads }}(t)\right)$

A centralized plan with the goal of reducing the charging prices of EV fleet comprising of several automobiles can be proposed, taking into account the forecasting of energy price data and driving details of EVs. The problem of optimization is conceived as a problem of binary linear programming, and solved using MOPSO. One can express the overall goal function as follows:

$\min f_{2}=\sum_{d=1}^{D} \sum_{n=1}^{N}\left(c_{d} \times P_{C h, n} \times S_{d, n} \times \Delta t\right)$

Where to go

Where PL, h represents hour of the main load.

The problem of optimization is subject to the limitations, such as charging rate, battery state and operating limits of the power system. The optimum charging plan for the EV battery would take advantage of the proximity of the EVs at the consumer contact point or the potential hours of operation for charging the EVs. In order to determine the possible EV market, the quality of the EV must be reviewed every hour. The energy consumption of the EVs to be used in the analysis depends o amount of EVs linked to power grid, the load efficiency, the SOCmax and the load SOC while the customer is coming home from the last trip (SOCinitial). The SOC of every EV battery is within the boundaries of each simulation phase:

$S O C_{i, \min } \leq S O C_{i}(h) \leq S O C_{i, \text { max }}$

To order to raise the efficacy of power network, the need for peak load following the introduction of EV charging/discharge energy to the market for domestic electricity must not be surpassed. Depending on the grid voltage power, the power intensities in busses are not allowed to fall below extreme:

$V_{i, \min } \leq V_{i}^{h} \leq V_{i, \max }$

In optimization process, the thermal restrictions of the system components correspond to the ratio of apparent flow of air, while the lines must be taken into account.

\section{THE PROPOSED MOPSO IMPLEMENTATION}

MOPSO is a family of computational optimization models designed to tackle both limited and unrestricted optimization issues. 
MOPSO is based on normal social actions and can be observed from working operators that are obviously inspired by a stylized depiction of movements of animals, for example, a bird flock or a fish classroom. One of MOPSO's main advantages is that it operates with set of potential solutions, named population, which can be updated by any generation of algorithms. In having a particle population, the algorithm is turned into a new generation of individuals. In the search-space, these particles are driven by a few basic formulas. Particle motions are driven by their top recognized quest place and best-known position of the entire swarm. Once improved locations are established, the motions of the swarm will be guided. The primary advantages of MOPSO over conventional optimization algorithms are that starting a search process does not require initial knowledge of the system, since they only work with particle coding, which is configured giving the objective purposes and the suitable constraints. In addition, the MOPSO algorithm can test several population points with iterative characteristics simultaneously within the search space. It is one of the most important differences from conventional optimization algorithms, where only one path can be taken in the search space. For all the particles, the strongest particles are selected, making system more expected to meet to a global optimum. MOPSO was chosen in this paper because they provide a great deal of versatility when considering multi-objective functions and can work with many constraints at the same time. In addition, as seen in this article, MOPSO has proved to be a robust optimization method for non-linear difficulties. The goal of the current analysis is to lessen variance of load profile by way of maximal EV charging. The hourly specifications are the variables used in the optimization problem. With this technique, MOPSO can handle 24-hour load requirements. Particles are initialized by real numbers corresponding to the total demand per hour. Hourly load requirement includes hourly house power consumption, hourly energy need from the grid to the vehicle system, and hourly EV power output from the vehicle to the grid system. The MOPSO engine is a multi-objective or fitness function that assigns importance to any individual in the residents and is used to push evolution procedure. In the document, the goals should satisfy the changing needs of much linked EVs at every hour and heterogeneity of load profile (residential demand for electricity or preload profile + EV load profile + power loss). During each hour, the MOPSO decides on the optimum demand for EV loads. The algorithm scheme is shown in Figure 3. Implementation of MOPSO starts with the initialization of the particlesThe 24-hour parking period, the 24-hour average EV requirement and the 24-hour demand for load are used as constraints for determining the potential for a viable solution for the region. As the starting population, a large sample size (100 people) is used to maximize the convergence rate towards a global solution. Community size selection is a key factor in MOPSO acceptance, as high values boost the convergence rate for a geographic solution. But this also improves measuring distance, too. Information of the topology of low voltage grids is used in the assessment process when planning an initial population. The feasibility of each entity in terms of multi-objective functions is assessed using a power flow, taking into account the constraints of the network. The cycle continues until the conditions for a stoppage have been met. Ultimately, the MOPSO result indicates the optimum EV charging power. The optimum solution is the pre-operational load plus electric vehicle input and the expected power loss in current surge.

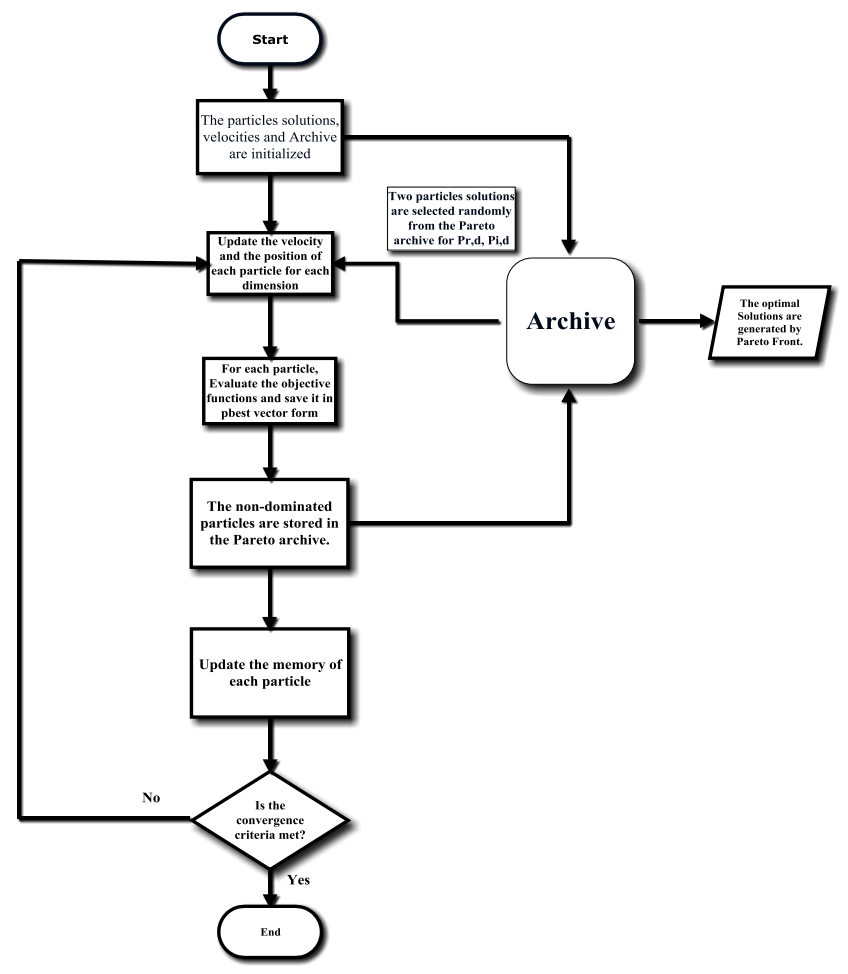

Figure 3. Flow chart of the MOPSO algorithm

\section{RESULT AND DISCUSSION}

Depending on each individual EV driver, the ability to predict the mobility behaviour of the EV is a major challenge in integrating EV into distribution power networks. The overall demand for EV charging is satisfied by a parking capacity plan needs to be developed in such mode that increases the capacity profile, which allows the smart network aggregator to decide on the future timing of the charging of EV. This paper focuses emphasis on times relevant to the research that lead to most trips. The regular day trips will be divided into following groups: (1) journeys to/from house, (2) travels to/from job, (3) vacation tours, (4) shopping tours, (5) accompanying tours, (6) tour to/from college (7) outings to friends, and (8) other trips. Through collecting trips from / to home, it is probable to evaluate the daytime the $\mathrm{EV}$ is plugged in at house. To determine the parking outline of a residential system for every 24 hours a day, the fraction of EVs that can be parked at house can be used. This is connected to the whole amount of network-connected EVs available. Figure 4 illustrates the pattern in suburban parking where all EVs are parked at 1:00 at home, while only 2 per cent of EVs are parked at 10:00 at home. Most of the EVs are mostly parked at house from $8 \mathrm{pm}$ until $7 \mathrm{am}$. Assuming most drivers leave home between the hours of 8 a.m. Up to 10 a.m. That corresponds to starting business, and returning home between 7 p.m. and instead, midnight. Figure 5 specifies the sum of hours in charging facility throughout the day while cars are plugged in. The hourly electricity generated via wind turbine for a day, and the solar power is considered from 5 A.M. in a daytime. Till 6 p.m.

Published By:

Blue Eyes Intelligence Engineering and Sciences Publication (C) Copyright: All rights reserved.

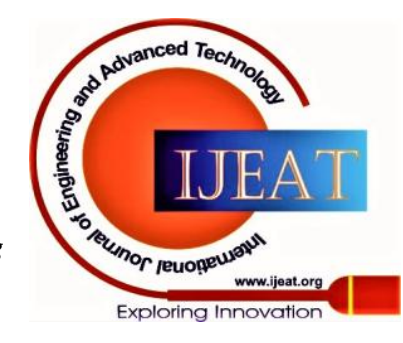


The power produced through the renewable source (airstream and solar) is indicated in Fig. 6. The power produced through the wind generator is noteworthy than the energy produced by the PV module. If the electricity provided by the renewable source is less than the demand, the controller will automatically link the grid and handle the power requirement. Around the same time, if the renewable plant generates more electricity than the planned demand then it must transfer the power to the grid.

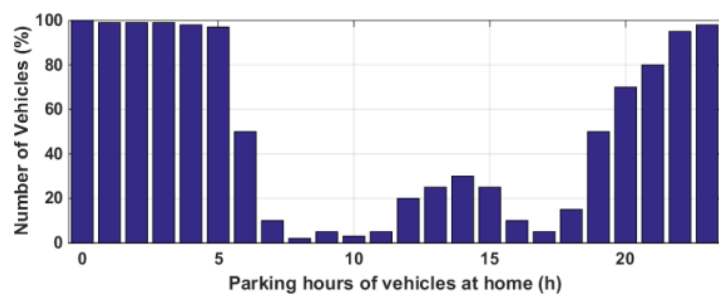

Figure 4. Residential parking pattern.

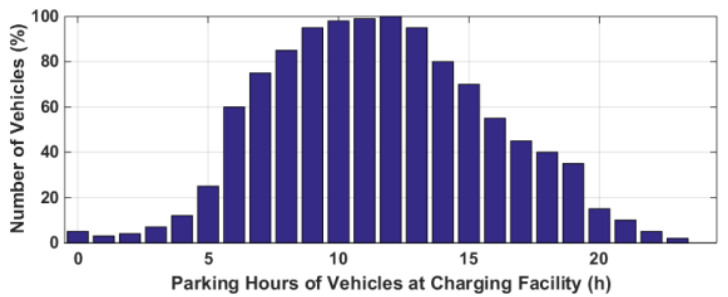

Figure 5. Parking hours of vehicles at charging facility.

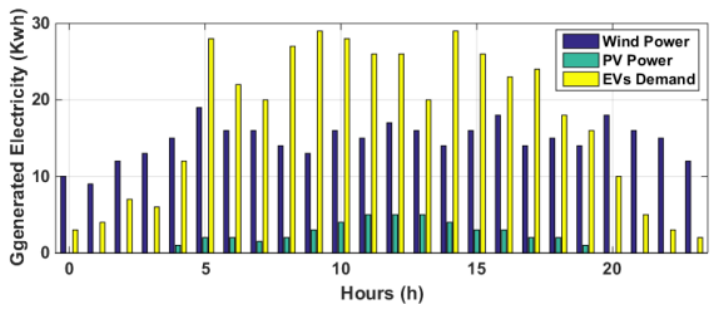

Figure 6. Hourly generated electricity from the proposed system, from both wind and solar.

The proposed optimum scheduling method for MOPSO had been executed in the suburban system. The residential network (Unregulated Charging Strategy, Conventional Controlled Charging, and MOPSO) had three separate forms of EV charging processes implemented. The first matches uncontainable, or uncontrolled, organization in which batteries are charge by EVs at home as they reach. The second fee structure is focused on Modern Regulated Charging. The third method coincides with the application of strategic management methods using MOPSO. The judgment variables in the proposed model are the wind structure's generated power, the power formed by PV units, the amount of batteries and the hourly capacity of the battery energy storing structure and hourly power of grid electricity purchase / sales. It must be distinguished that MOPSO-based management charging strategies increase the capacity profile and overall load demand during valley time frames. This is accomplished by the EVs' capacity of discharging their batteries and supplies this to the power grid, therefore decreasing the power grid burden. In the proposed Smart MOPSO, when parked at home, electric vehicles are to be supplied with tools capable of supplying energy stored in the battery to the network. Thus, EVs would perform as generators and chargers. This new capability, by functioning as versatile energy storage infrastructure permits EVs to provide ancillary facilities for example spinning resources and control to network operatives. Intelligent scheduling with a mix of grid-to-vehicle and grid-to-vehicle provides the opportunity to handle EVs as a grid-to-grid vehicle with a smart grid aggregator providing maximum savings. These can also be used as a grid for vehicle vendors, providing valley filling facilities. The capacity of EVs to serve as versatile storage systems could be controlled in relations of energy necessities for regular travel and energy storage requirements as a manufacturer. The storage capacity can pay for a greater integration of distributed energy reserves by assessing their energy variability. Grid-based EVs can be powered, discharged, or disconnected at any time of day. The maintenance system would automate the charging and discharge of the batteries to maximize the operation of the power grid. In the case of smart MOPSO projects, a proportion of the energy contained in $\mathrm{EV}$ batteries is required to be supplied to the grid multiple hours a day. Two timeframes are considered: daytime (7:00-18:59), when 10 per cent of the electricity provided by EVs can be injected into the network, and at night when 90 per cent of EVs are plugged in at home. We're able to move any of their accumulated energy to the net. When a power grid operator does not monitor the charging actions of EVs, the charging method can be referred to as unregulated charging. Electric vehicles shall be paying with an uncontrolled charging policy as soon as the EV is plug-in. For these uncoordinated charging cases, personal expectations and other unpredictable variables influence the initial charging period and the charging time. The main aim of charging is to provide enough energy to meet travel needs, and the owner of the EV does not recognize the influence of charging on the power network. It can be concluded, giving the possibility limit theorem that the first charging period for EVs is at normal distribution. When people typically plug EVs into the charging grid while they get house from job, overall charges rise exponentially at 18:00, which is the topmost period of energy use in uncoordinated charging scenarios, thus uncoordinated charging raises a peak valley gap between 18:00 and 21:00. The EVs are deemed to be linked to the system and are thus charged at the subsequent times. $85 \%$ of the EVs reach at house between 5.30 p.m. and 8.30 p.m. and are to be automatically hooked into the energy grid. This time period happens for the duration of the greatest load demand, which is called a peak season. Five per cent of EVs are linked to the system between 24:00 and 3:00 that correlates to the demand for valley load. Between 21:00 to $24: 00,10 \%$ of EVs are charged for. Figure 7 indicates the percentage of residential network load on uncontrolled EV charging cycle. It should be recalled that main substation is overwhelmed at peak times with "dumb charging," which corresponds to a 140 per cent overload at 20:00. The EV will be hooked into the power grid for traditional regulated charging as long as the driver reaches at charging facility / home. The charging cycle is then controlled on a time-frame basis by the smart grid operator.. For this case, two time ranges are considered for the average daytime charge of EVs: $10 \%$ of parked EVs are charged for period of daytime (7:00-18:59) while $90 \%$ of EVs are charged at night time (18:30-5:54).

Published By:

Blue Eyes Intelligence Engineering and Sciences Publication 
Figure 8 shows the final burden (percentage) using traditional managed charging. The EV load control strategy increases the post-operation load profile of the power grid, as presented in Fig 8 . In this scenario, the peak load should not be reached at any hour. It can be found that the market for power is spread all night, so that there is no lack of energy. The smart MOPSO load managing method enhances the burden outline of the utility grid after service, as shown in Figure 9. Due to the V2 $\mathrm{G}$ features of the $\mathrm{EV}$, it is possible to reduce the load requirements on power grid marginally during the daytime. The load profile is then shortened by $20 \%$ between $24: 00$ and 6:00 according to the total load frequency for the G2V central transformer network. This should be noted, as in the previous example, that the smart MOPSO strategy avoids overloading and determines parking prices. Figure 10 displays the power profile through feeder cables where EVs are linked to different charging strategies: uncontrolled charging, traditional charging and smart MOPSO management. It should be remembered that, when using smart MOPSO storage, power flows across feeder lines are relatively steady throughout the day. By charging each EV, the peak load is $1,275 \mathrm{PU}$, the valley load (minimum) is 0,2049 PU, and the peak-to-valley discrepancy is $0,90635 \mathrm{PU}$, compared to 1,008 PU, 0,5295 PU and 0,4928 PU, respectively, with maximum loading and unloading of EVs. As seen, the synchronized charging and unloading technique for EVs in the power valleys and peaks is more effective than no uncoordinated charging EVs. The distributed loss curves of the network for the different charging methods as seen in Fig. 11. The average one-day transmission deficit is $0.6298 \mathrm{KW}$ with no EVs attached to the power grid, opposed to $0.9085 \mathrm{KW}$ when the EVs are uncoordinated. The gross transmission loss is $0.40934 \mathrm{KW}$ after the power grid operator monitors the charging and discharge of EVs using an optimal charging system. As a result, transmission losses are limited to 27,423 per cent compared to the uncoordinated carrying loss.

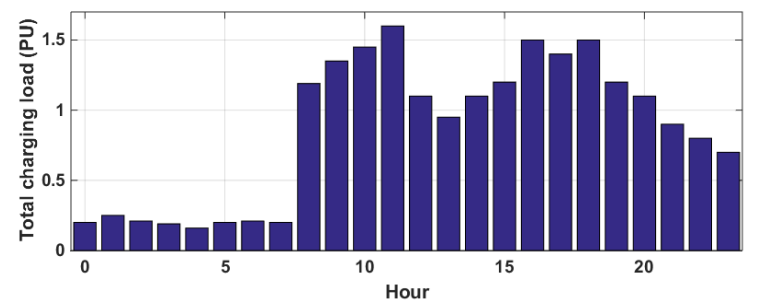

Figure 7. Total load curve of EVs by uncoordinated charging schedule.

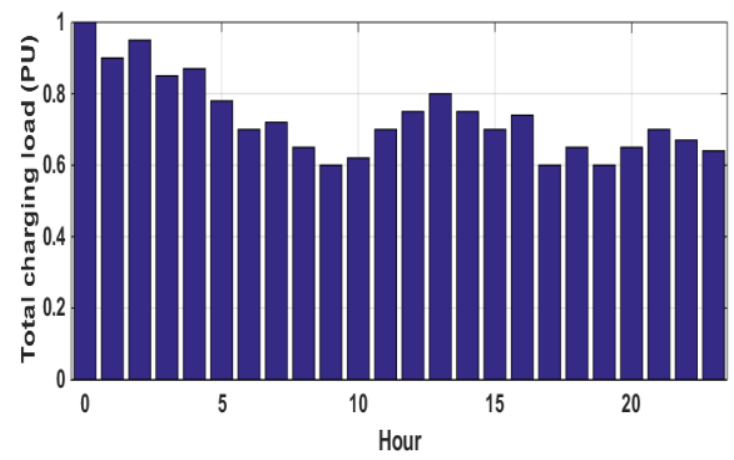

Figure 8. Total charging load curve using conventional controlled charging.

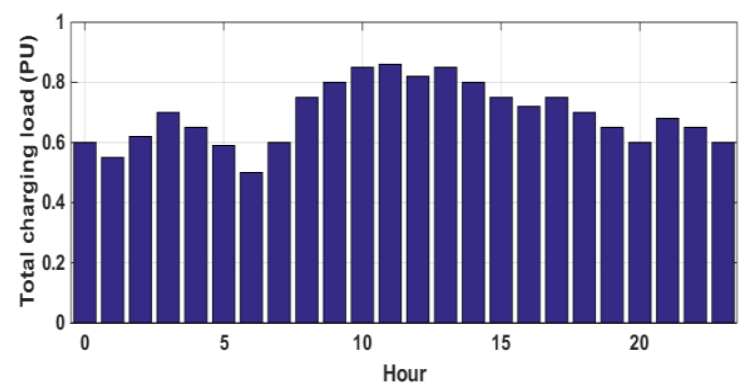

Figure 9. Total charging load curve by MOPSO.

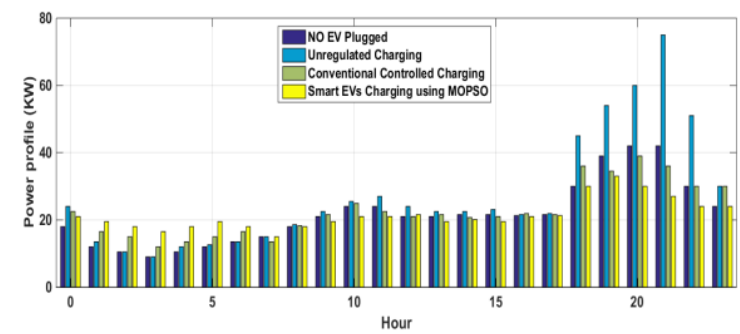

Figure 10. Power profile with various charging strategies.

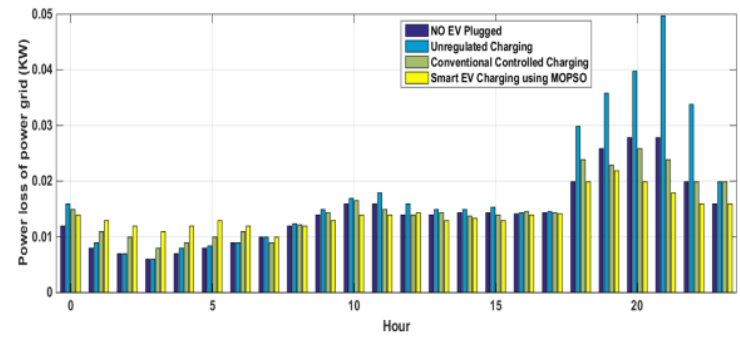

Figure 11. Power-loss power system for various charging methods.

\section{CONCLUSION}

This paper suggested an integrated network of charging station for electric vehicles, battery energy storing structure and Wind / PV hybrid structure to efficiently increase the utilization rate of wind and renewable solar energy resources. So address the issue of $\mathrm{EV}$ charging stations requiring difficult access to the power grid. An optimal scheduling strategy for electric vehicles (EVs) charging in MOPSO-based supply networks was built to smooth the day-to-day power demand curve and assess the discharging/ charging arrangement of EV batteries and to interchange energy with the service grid. To automate the charging and discharge of a great amount of EVs in the power network, The MOPSO is designed to boost the economic and technological efficiency of power network operations. Also reduce the negative effect as a result of a great amount of PHEVs linked to the power network. Outcomes of the model study displayed that the plan would increase the self-consuming degree of wind / PV production, reduction in highest electricity depletion throughout charging of EV and the EV charging price. The optimisation approach is beneficial in resolving the impact on grid problems of large-scale supplied power assimilation and charging of EV. With good stability efficiency, the proposed approach can handle the multi-objective optimization problem well, and can provide Pareto solutions for decision makers.

Published By:

Blue Eyes Intelligence Engineering and Sciences Publication

(C) Copyright: All rights reserved.

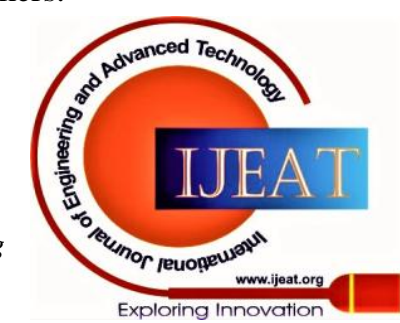




\section{REFERENCES}

1. Saxena Samveg, Gopal Anand, Phadke Amol. Electrical consumption of two-, three- and four-wheel light-duty electric vehicles in India. Appl Energy 2014;115:582-90.

2. Fathabadi, H. Novel grid-connected solar/wind powered electric vehicle charging station with vehicle-to-grid technology. Energy 2017, 132, 1-11.

3. Brouwer Anne Sjoerd, Kuramochi Takeshi, Broek Machteld van den, Faaij André. Fulfilling the electricity demand of electric vehicles in the long term future: an evaluation of centralized and decentralized power supply systems. Appl Energy 2013;107:33-51.

4. Foley Aoife, Tyther Barry, Calnan Patrick, Gallachóir Brian Ó. Impacts of electric vehicle charging under electricity market operations. Appl Energy 2013;101(January):93-102.

5. Khayyam Hamid, Abawajy Jemal, Javadi Bahman, Goscinski Andrzej, Stojcevski Alex, Bab-Hadiashar Alireza. Intelligent battery energy management and control for vehicle-to-grid via cloud computing network. Appl Energy 2013;111:971-81.

6. Sorrentino Marco, Rizzo Gianfranco, Sorrentino Luca. A study aimed at assessing the potential impact of vehicle electrification on grid infrastructure and road-traffic green house emissions. Appl Energy 2014;120:31-40

7. Han Sekyung, Han Soohee, Aki Hirohisa. A practical battery wear model for electric vehicle charging applications. Appl Energy 2014;113:1100-8.

8. Zhang, P.; Qian, K.; Zhou, C.; Stewart, B.G.; Hepburn, D.M. A Methodology for Optimization of Power Systems Demand Due to Electric Vehicle Charging Load. IEEE Trans. Power Syst. 2012, 27, 1628-1636.

9. Zheng, J.; Wang, X.; Men, K.; Zhu C.; Zhu, S. Aggregation Model-Based Optimization for Electric Vehicle Charging Strategy. IEEE Trans. Smart Grid 2013, 4, 1058-1066.

10. Zheng, J.; Wang, X.; Men, K.; Zhu C.; Zhu, S. Aggregation Model-Based Optimization for Electric Vehicle Charging Strategy. IEEE Trans. Smart Grid 2013, 4, 1058-1066.

11. Luo, Z.; Hu Z.; Song, Y.; Xu, Z.; Lu, H. Optimal Coordination of Plug-In Electric Vehicles in Power Grids With Cost-Benefi Analysis-Part I: Enabling Techniques. IEEE Trans. Power Syst. 2013, 28, 3546-3555.

12. P. Rezaei, J. Frolik, and P. Hines, "Packetized plug-in electric vehicle charge management," Smart Grid, IEEE Transactions on, vol. 5, no. 2 , pp. 642-650, March 2014.

13. Trovão João P, Pereirinha Paulo G, Jorge Humberto M, Antunes Carlos Henggeler. A multi-level energy management system for multi-source electric vehicles - an integrated rule-based meta-heuristic approach. Appl Energy 2013;105(May):304-18.

14. He Yifeng, Venkatesh Bala, Guan Ling. Optimal scheduling for charging and discharging of electric vehicles. IEEE Trans Smart Grid 2012;3(3):1095-2015.

15. Jin Chenrui, Tang Jian, Ghosh P. Optimizing electric vehicle charging with energy storage in the electricity market. IEEE Trans Smart Grid 2013;4(1):311-20.

16. Kristoffersen Trine Krogh, Capion Karsten, Meibom Peter. Optimal charging of electric drive vehicles in a market environment. Appl Energy 2011;88(5):1940-8.

17. Sortomme Eric, El-Sharkawi Mohamed A. Optimal scheduling of vehicle-to-grid energy and ancillary services. IEEE Trans Smart Grid 2012;3(1):351-9.

18. Cao Yijia, Tang Shengwei, et al. An optimized EV charging model considering TOU price and SOC curve. IEEE Trans Smart Grid 2012;3(1):388-93

19. Sundström Olle, Binding Carl. Flexible charging optimization for electric vehicles considering distribution grid constraints. IEEE Trans Smart Grid 2012;3(1):26-37.

20. Esfandyari, A.; Norton, B.; Conlon, M.; McCormack, S.J. Performance of a campus photovoltaic electric vehicle charging station in a temperate climate. Sol. Energy 2019, 177, 762-771.

21. Chaudhari, K.; Ukil, A.; Kumar, K.N.; Manandhar, U.; Kollimalla, S.K. Hybrid Optimization for Economic Deployment of ESS in PV-Integrated EV Charging Stations. IEEE Trans. Ind. Inform. 2018, 14, 106-116.

22. Bhatti, A.R.; Salam, Z.; Sultana, B.; Rasheed, N.; Awan, A.B.; Sultana, U.; Younas, M. Optimized sizing of photovoltaic grid-connected electric vehicle charging system using particle swarm optimization. Int. J. Energy Res. 2019, 43, 500-522.
23. Badea, G.; Felseghi, R.-A.; Varlam, M.; Filote, C.; Culcer, M.; Iliescu, M.; Răboacă, M.S. Design and Simulation of Romanian Solar Energy Charging Station for Electric Vehicles. Energies 2019, 12, 74.

24. Domínguez-Navarro, J.A.; Dufo-López, R.; Yusta-Loyo, J.M.; Artal-Sevil, J.S.; Bernal-Agustín, J.L. Design of an electric vehicle fast-charging station with integration of renewable energy and storage systems. Int. J. Electr. Power Energy Syst. 2019, 105, 46-58.

25. Torreglosa, J.P.; García-Triviño, P.; Fernández-Ramirez, L.M.; Jurado, F. Decentralized energy management strategy based on predictive controllers for a medium voltage direct current photovoltaic electric vehicle charging station. Energy Convers. Manag. 2016, 108, 1-13.

26. Liu, J.; He, D.; Wei, Q.; Yan, S. Energy Storage Coordination in Energy Internet Based on Multi-Agent Particle Swarm Optimization. Appl. Sci. 2018, 8, 1520

\section{AUTHORS PROFILE}

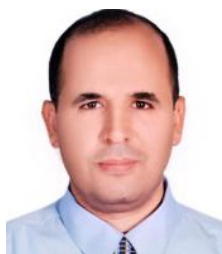

Adel Elgammal is currently an Associate Professor at the University of Trinidad and Tobago UTT, Utilities Engineering. He received his B.Sc. Degree in Electrical Power Engineering from Helwan University-EGYPT in 1996. He completed his M.Sc Degree in Electric Drives and Machines Engineering in 2002 and Ph.D. Degree in Jan-2007 from the Faculty of Engineering (Helwan University-EGYPT) Dr. Elgammal authored and co-authored over 65 Scholarly Technica Journals, and over 77 Refereed Conference Publications and three Engineering Book Chapters.

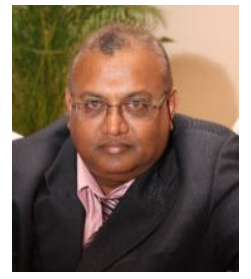

Dr. Tagore Ramlal is currently an Assistant Professor in Utilities Engineering, University of Trinidad and Tobago. He has been involved in the field of training and development in Electrical Engineering with diversified experience of thirty- six years (36) at the tertiary level. His contribution towards professional outreach program spans twenty-three (23) years with expertise in consultation, development and facilitation of specialized industry courses. He also has extensive experience (16) years in electrical engineering design and consultancy services. Tagore has been employed with University of Trinidad and Tobago since 2000.

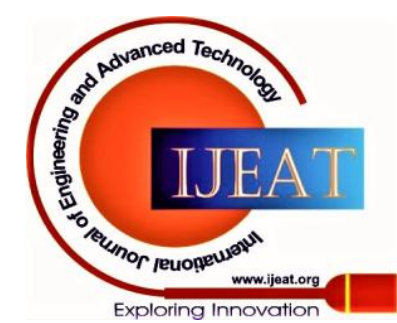

